Poincare Journal of Analysis E Applications

Vol. 2015 (2), Special Issue (IWWFA-II, Delhi), 93-103

(C) Poincare Publishers

\title{
A NEW SPLITTING TRICK FOR WAVELET PACKETS ON LOCAL FIELDS OF POSITIVE CHARACTERISTIC
}

\author{
Firdous A. Shah ${ }^{\dagger}$ and M. Younus Bhat \\ Date of Receiving : $\quad$ 24.02. 2015 \\ Date of Revision : 12.03. 2015 \\ Date of Acceptance : 15.03 .2015
}

\begin{abstract}
This paper deals with a construction of wavelet packets on local fields of positive characteristic. The splitting trick here is different that the method described by Behera and Jahan in [Wavelet packets and wavelet frame packets on local fields of positive characteristic, J. Math. Anal. Appl. 395 (2012), 1-14], in that we split the wavelet subspaces directly instead of using the low-pass and high-pass filters associated with the multiresolution analysis. Furthermore, the method overcomes the difficulty of constructing non-orthogonal wavelet packets of the dilation factor not equal to 2 .
\end{abstract}

\section{Introduction}

A field $K$ equipped with a topology is called a local field if both the additive $K^{+}$ and multiplicative groups $K^{*}$ of $K$ are locally compact Abelian groups. The local fields are essentially of two types: zero and positive characteristic (excluding the connected local fields $\mathbb{R}$ and $\mathbb{C}$ ). Examples of local fields of characteristic zero include the $p$ adic field $\mathbb{Q}_{p}$ where as local fields of positive characteristic are the Cantor dyadic group and the Vilenkin $p$-groups. Even though the structures and metrics of local fields of zero and positive characteristics are similar, their wavelet and multiresolution analysis theory are quite different. In recent years, local fields have attracted the attention of several mathematicians, and have found innumerable applications not only to number theory but also to representation theory, division algebras, quadratic forms and algebraic geometry. As a result, local fields are now consolidated as part of the standard repertoire of contemporary mathematics. For more about local fields and their applications, we refer to the monographs $[12,23]$.

In recent years there has been a considerable interest in the problem of constructing wavelet bases on various groups, namely, Cantor dyadic groups [10], locally compact Abelian groups [7], $p$-adic fields [9] and Vilenkin groups [11]. Recently, R. L. Benedetto and J. J. Benedetto [2] developed a wavelet theory for local fields and related groups.

2010 Mathematics Subject Classification. 42C40, 42C15, 43A70, 11 S85.

Key words and phrases. Wavelet, multiresolution analysis, splitting trick; wavelet packet, Riesz basis, local field, Fourier transform.

Communicated by. Yuri A. Farkov

${ }^{\dagger}$ Corresponding author. 